\title{
Delimitação de sítios ambientais homogêneos no Estado do Espírito Santo, com base no relevo, solo e clima
}

\author{
Definition of environmental sites in Espírito Santo state based on relief, soil and climate
}

\author{
Yhasmin Paiva Rody ${ }^{\mathrm{I}}$ André Quintão de Almeida ${ }^{\mathrm{I}}$ Aristides Ribeiro $^{\mathrm{II}}$ \\ Gilberto Chohaku Sediyama' ${ }^{\mathrm{I}}$ José Eduardo Macedo Pezzopane ${ }^{\mathrm{III}}$
}

\section{RESUMO}

\begin{abstract}
Este trabalho teve como objetivo definir sitios ambientais homogêneos no Estado do Espirito Santo, a fim de indicar o uso potencial do solo de todo o seu território. Técnicas estatísticas multivariadas foram empregadas sobre informações do relevo, coordenadas geográficas, dados do solo e clima. A disponibilidade hídrica e a evapotranspiração real e potencial foram determinadas a partir do resultado do balanço hídrico climatológico. Inicialmente, foi executada a análise de componentes principais (ACP) sobre os dados climáticos e, em seguida, a de agrupamento nas informações do relevo, solo, nos escores climáticos provenientes da ACP e nas coordenadas geográficas. Os dois primeiros eixos da ACP explicam cerca de $85,3 \%$ da variância total acumulada. Após a rotação dos componentes principais, as melhores variáveis correlacionadas ao primeiro eixo foram a amplitude térmica, a temperatura média anual, seguida da evapotranspiração real. Foram definidos seis sitios ambientais para o Estado do Espirito Santo e alguns apresentaram similaridades ao clima. As variáveis topográficas foram os fatores de maior influência na diferenciação dos sitios. A metodologia se mostrou adequada na definição dos sítios.

Palavras-chave: zoneamento climático, estatistica multivariada, balanço hídrico.
\end{abstract}

\section{ABSTRACT}

The objective of this study was to define homogeneous environmental sites in the state of Espirito Santo, Brazil which may possibly be used to indicate potential soil use for the entire state. Multivariate statistics techniques were employed using information of the relief and geographical coordinates, as well as soil and climatic data. Water availability and real and potential evapotranspiration were determined from the results of the climatological water balance. Initially a principal component analysis (PCA) for the climatological data was performed. Subsequently, a grouping analysis was executed on the relief, soil and climatic data provided by the PCA and geographical coordinates. The first two axes of the PCA define roughly $85.3 \%$ of the total accumulated variance. After rotation of the principal components, the variables that best correlated to the first axis were the thermal amplitude, average annual temperature, followed by real evapotranspiration. Six environmental sites were defined for the state of Espirito Santo, some presenting climatic similarities. The topographic variables were the factors of greatest influence on the sites differentiation. The methodology was appropriate in defining the sites.

Key words: climate zoning, multivariate statistics, water balance.

\section{INTRODUÇÃO}

O Estado do Espírito Santo destaca-se no cenário agrícola nacional, com diversificada cadeia agrícola de produção. É grande produtor de café, eucalipto de fibra curta, frutas, em especial o mamão papaya, algumas hortaliças, dentre outros cultivos. Com distintas condições físico-climáticas, o Estado apresenta, em sua porção norte, clima quente e seco; na região central, frio e úmido; ao sul, caracterizado por calor e alta umidade. Essa variedade de ambientes favorece a diversificação agrícola, facilitando a implantação de diversas culturas, cada qual distribuída em regiões específicas.

'Programa de Pós-graduação em Meteorologia Agrícola, Departamento de Engenharia Agrícola, Universidade Federal de Viçosa (UFV), Viçosa, MG, Brasil.

"Departamento de Engenharia Agrícola, UFV, 36570-000, Viçosa, MG, Brasil. E-mail: ribeiro@ufv.br. Autor para correspondência. "IIPrograma de Pós-graduação em Ciência Florestal, Departamento de Engenharia Florestal. Universidade Federal do Espírito Santo (UFES), Alegre, ES, Brasil. 
A delimitação das diferentes regiões passa pela identificação de áreas homogêneas e pelo zoneamento, que visa conhecer as características físicas (clima, solo, relevo), biológicas (fauna, flora) e socioeconômicas (presença e atividades desenvolvidas pelo homem).

Com base na fertilidade do solo, ORTEGA \& SANTIBÁÑEZ (2007) individualizaram zonas de manejo para o cultivo de milho (Zea mays $\mathrm{L}$ ) na região central do Chile. LYRA et al. (2006) delimitaram regiões homogêneas para a província do Táchira na Venezuela, baseadas na sazonalidade da precipitação pluvial mensal e na distribuição de probabilidade das chuvas. GRAEF et al. (2005) determinaram ecorregiões para serem usadas em estudos de redes de monitoramento ambiental para Brandenburg, Alemanha.

Na grande maioria desses estudos, técnicas de análise estatística multivariada são empregadas (DINPASHOH et al., 2004; TIMILSENA\& PIECHOTA, 2008; HARTMANN \& WUNNEMANN, 2009). MORRIS et al. (2009) realizaram a geoespacialização por meio de dados climáticos e de relevo, utilizando a Análise de Componentes Principais (ACP). Hoje em dia, as geotecnologias (sensoriamento remoto, sistemas de informações geográficas) também são utilizadas nesse tipo de análise (RHEE et al., 2008; GUILLEMETTE et al., 2009).

Com base no exposto, este trabalho teve como objetivo definir sítios ambientais homogêneos para o Estado do Espírito Santo, a fim de definir o uso potencial do solo, utilizando técnicas de estatísticas multivariadas, a partir de informações de relevo, solo, as coordenadas geográficas e clima.

\section{MATERIAL E MÉTODOS}

O local do estudo compreende o Estado do Espírito Santo, situado entre as coordenadas de $-17^{\circ} 52^{\prime}$ e $-21^{\circ} 17^{\prime}$ de latitude e $-41^{\circ} 54^{\prime}$ e $-39^{\circ} 38^{\prime}$ de longitude, com área de $46077,51 \mathrm{~km}^{2}$ (Figura 1-A). O Estado apresenta três regiões de distintos padrões de relevo: Litorânea; Tabuleiros costeiros; e Elevada do Interior e a área enquadra-se nas zonas climáticas $\mathrm{A}$ e C (classificação de Koppen), sendo encontrado os subtipos climáticos Aw, Am, Cfe Cw.

Para alcançar os objetivos propostos, foram utilizados dados do Instituto Brasileiro de Geografia e Estatística (IBGE), da Empresa Brasileira de Pesquisa Agropecuária (EMBRAPA) e da Agência Nacional das Águas (ANA). Com o auxílio de um Sistema de Informação Geográfica (SIG), essas informações foram preparadas para as análises estatísticas multivariadas.
Primeiramente, foi construída uma malha digital georreferenciada de $1 \mathrm{~km} \times 1 \mathrm{~km}$. Para cada célula da malha, foram obtidas as respectivas latitude e longitude centrais.

As características do relevo foram extraídas das cartas topográficas planialtimétricas do IBGE. Para isso, foi produzido um Modelo Digital de Elevação (MDE), interpolando as curvas de nível pelo método do Inverso do Quadrado da Distância (IDW), com expoente 2. Com o MDE gerado, os valores de altitude, declividade e face de exposição foram calculados e associados à mesma malha de $1 \mathrm{~km} \mathrm{x} 1 \mathrm{~km}$. A caracterização do solo foi realizada pela sua disponibilidade total de água (DTA), a partir do mapa de tipo de solo disponibilizado pela EMBRAPA. Esse mapa foi importado para o ambiente SIG. A variação da DTA foi calculada para cada célula da malha de $1 \mathrm{~km}$ pelo produto entre a densidade de cada classe de solo e a diferença entre sua Capacidade de Campo $(\mathrm{Cc})$ e seu Ponto de Murcha (Pm). De acordo com a classe de textura de cada solo, os valores de Cc (\% em peso), $\mathrm{Pm}(\% \mathrm{em}$ peso $)$ e densidade $\left(\mathrm{g} / \mathrm{cm}^{3}\right)$ usados foram os sugeridos por BERNARDO et al. (2005).

Os valores de precipitação foram obtidos em dados anuais médios, a partir dos 86 postos

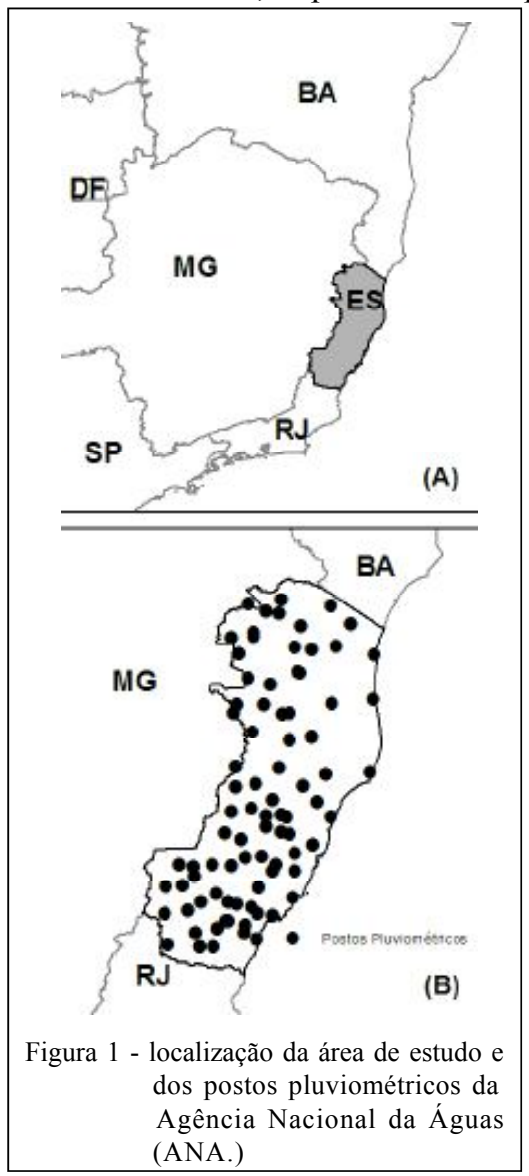

Ciência Rural, v.40, n.12, dez, 2010. 
pluviométricos da Agência Nacional de Águas (ANA), inseridos na área de estudo (Figura 1-B), considerando o período de 1976 a 2008.

Para a estimativa da temperatura média, utilizou-se o modelo desenvolvido por PEZZOPANE et al. (2004) para o Estado do Espírito Santo. Nesse modelo, foram aplicadas, como variáveis de entrada, a altitude e a latitude, que apresentam maior efeito na estimativa da temperatura. Para o cálculo da amplitude térmica mensal, apresentaram-se as mesmas variáveis do modelo anterior, acrescidas da variável longitude, em modelos desenvolvidos por SEDIYAMA et al. (2002) para a estimativa das temperaturas mínimas e máximas no Estado do Espírito Santo.

O método de THORNTHWAITE \& MATTER (1955) foi utilizado no cálculo do balanço hídrico climatológico, tendo como variáveis de entrada os dados médios mensais de temperatura e precipitação. A evapotranspiração potencial foi estimada pelo método de THORNTHWAITE (1948), que utilizou os dados de temperatura média mensal. Por meio desses cálculos, foram obtidos a evapotranspiração real, a deficiência e o excedente hídrico. A capacidade máxima adotada de armazenamento de água no solo (CAD) foi a climatológica (100mm).

A duração do dia ou o fotoperíodo foi calculado para os solstícios de inverno e verão, visto que estes representam os dias do ano com a menor e maior duração, respectivamente. Utilizaram-se as equações apresentadas por IQBAL (1983), com estimativas realizadas a partir de: ângulos horários, correspondentes à meia varredura da trajetória do Sol acima do horizonte; declinação solar, que equivale à posição relativa do Sol sobre a superfície da Terra; e do dia Juliano correspondente.

Para se obter uma combinação dos componetes do clima anteriormente citados, realizouse a ACP. A fim de encontrar uma matriz de pesos mais facilmente interpretáveis, foi feita a rotação ortogonal dos fatores por meio do método varimax. Os escores climáticos ortogonais foram interpolados por meio do Inverso do Quadrado da Distância (IQD).

Posterior a essa etapa, foi executada a análise de agrupamento sobre o relevo, os escores climáticos, provenientes da ACP, a disponibilidade total de água no solo e as coordenadas geográficas. A aplicação de modelos estatísticos multivariados foi fator dependente das unidades e das escalas em que as variáveis foram medidas. Diante das distintas magnitudes dos valores das variáveis apresentadas, realizou-se a normalização destes, através da diferença entre o valor observado e a sua respectiva média, em relação ao desvio-padrão da variável em questão. Foi empregada a técnica não hierárquica $K$-means cluster, com base na distância de Mahalanobis com o número inicial de nove sítios. O método teve o centróide como critério de agregação das respostas.

Ao final, foi verificada a consistência dos grupos estabelecidos pela análise de agrupamento, por meio da análise estatística discriminante. Os testes $\mathrm{F}$ e Wilks foram utilizados para testar a significância da análise.

\section{RESULTADOS E DISCUSSÃO}

Avaliando os autovalores e as porcentagens das variâncias, gerados a partir da ACP, verificou-se que os dois primeiros eixos explanam cerca de $85,3 \%$ da variância total acumulada. Ao realizar essa mesma análise em variáveis climáticas, RAZIEI et al. (2008) observaram 65,7\% de variância acumulada para os dois primeiros componentes.

O grupo de variáveis melhores correlacionadas ao primeiro eixo (CP1) é: ETP $(r=0,95)$, temperatura média $(\mathrm{r}=0,93)$ e, na parte negativa do primeiro eixo, o excedente hídrico $(\mathrm{r}=-0,90)$. A evapotranspiração real (ETR) foi a variável meteorológica melhor correlacionada ao segundo eixo (CP2) com r $=0,74$. Depois da rotação dos componentes principais (Tabela 1), verificou-se, para o primeiro componente, que as três variáveis melhores correlacionadas descritas na ACP são a temperatura média, amplitude térmica e a ETR. Para o segundo componente rotacionado, tem-se que a temperatura média e a ETR foram as mais correlacionadas.

Analisando as comunalidades do primeiro fator para o grupo de variáveis mais explicativas após a rotação, observou-se que todas apresentaram valores maiores que 0,8 , isto é, pelo menos $79 \%$ da variância total é explicada por quatro variáveis (amplitude térmica, temperatura média, ETR e ETP). Com exceção da precipitação, todas as variáveis apresentaram comunalidade em mais de $80 \%$ da variância total no segundo fator, indicando forte contribuição dessas.

Os valores de Wilks' Lambda calculados se aproximaram de zero, indicando maior grau de diferenciação entre os grupos. Satisfez-se a hipótese de nulidade, confirmando o bom desempenho da discriminação. O Teste " $F$ " $(P \leq 0,05)$ mostrou diferenças significativas entre as médias, sugerindo que as observações são derivadas de populações diferentes. Mesmo apresentando resultados satisfatórios, a análise discriminante identificou células agrupadas equivocadamente. Foram reagrupadas $9,2 \%$ de células das inicialmente agrupadas. 
Dos nove grupos formados inicialmente, alguns apresentaram semelhanças entre as características analisadas. Outro agrupamento foi realizado a partir das distâncias centrais dos nove sítios predefinidos, utilizando o método de agrupamento hierárquico linkage simples (vizinho mais próximo), em um processo sistemático para formação dos grupos. A partir do conhecimento prévio da área em estudo, verificou-se que o número de seis sítios apresentou coerência e evitou excessiva estratificação (Figura 2).

A caracterização físico-climática dos seis sítios ambientais homogêneos (SAH) é apresentada na tabela 2. Com base nesses resultados, é possível indicar o potencial uso do solo, de acordo com os aspectos de cada uma das regiões delimitadas. Devese ressaltar que foram abordadas nesta discussão as variáveis físico-climáticas que apresentaram diferenças significativas entre cada sítio.

Os SAHs 1 e 2 se localizam na porção norte e juntos compõem $50,58 \%$ da área do Estado. Foi observada uma grande região de transição, na qual não se define uma área contígua pertencente a um único sítio, evidenciando a semelhança entre eles. As características climáticas são semelhantes, com maiores déficits hídricos e temperaturas, em relação aos demais, com excedente hídrico menos expressivo.

Para o suprimento hídrico nessas áreas, técnicas de irrigação podem ser utilizadas. Na região norte, a qualidade climática aumenta a produtividade dos plantios irrigados e favorece o manejo da cultura do café Conilon (Coffea canephora), aumentando a sua produtividade e fazendo do Estado o maior produtor nacional dessa espécie (INCAPER, 2009).

Tabela 1 - Fração representada pelos autovalores ou as variâncias dos componentes principais em relação ao Traço (variância total).

\begin{tabular}{lcccc}
\hline Variáveis & \multicolumn{2}{c}{ Comunalidades } & \multicolumn{2}{c}{$\begin{array}{c}\text { Componentes } \\
\text { Rotacionados }\end{array}$} \\
& Fator 1 & Fator 2 & Fator 1 & Fator 2 \\
\hline Precipitação & 0,038 & 0,662 & $-0,195$ & $-0,790$ \\
Temperatura média & 0,826 & 0,970 & 0,909 & 0,380 \\
Amplitude térmica & 0,902 & 0,922 & 0,950 & 0,141 \\
ETP & 0,796 & 0,973 & 0,892 & 0,422 \\
ETR & 0,825 & 0,861 & 0,908 & $-0,190$ \\
Déficit hídrico & 0,194 & 0,859 & 0,440 & 0,816 \\
Excedente hídrico & 0,482 & 0,824 & $-0,694$ & $-0,585$ \\
Fotoperíodo de verão & 0,012 & 0,801 & $-0,107$ & $-0,889$ \\
Fotoperíodo de inverno & 0,012 & 0,801 & 0,107 & 0,889 \\
\hline
\end{tabular}

A diferenciação na delimitação das áreas homogêneas entre esses sítios foi determinada por fatores topográficos. Foram encontrados no SAH 1, menores altitudes e declividades em relação ao SAH 2. $\mathrm{O}$ relevo suave presente possui um grande potencial para aplicação de práticas mecanizadas, nas diversas fases da operação agrícola, e, consequentemente, um aumento da produtividade das operações de colheita (MACHADO, 2004).

O SAH 3 correspondeu a $17,94 \%$ da área do Estado do Espírito Santo. Esse ambiente apresentou altitudes mais elevadas e consequentemente menores temperaturas. Essas condições são somadas ao suprimento hídrico favorável, indicado pelo menor déficit hídrico. Nessas áreas, encontram-se plantios de espécies típicas de climas mais frios como morango, pêssego e uva. Observa-se a presença de pequenas áreas no interior e, sobretudo, no limite desse sítio, onde se encontrou a quarta área homogênea.

Com $5,75 \%$ da área total do Estado, o SAH 4 evidenciou as maiores declividades médias (topos de morros). Esse fato o torna pouco apto à exploração intensiva do solo, o que deve ser destinado a áreas de proteção ambiental, conforme a legislação brasileira vigente.

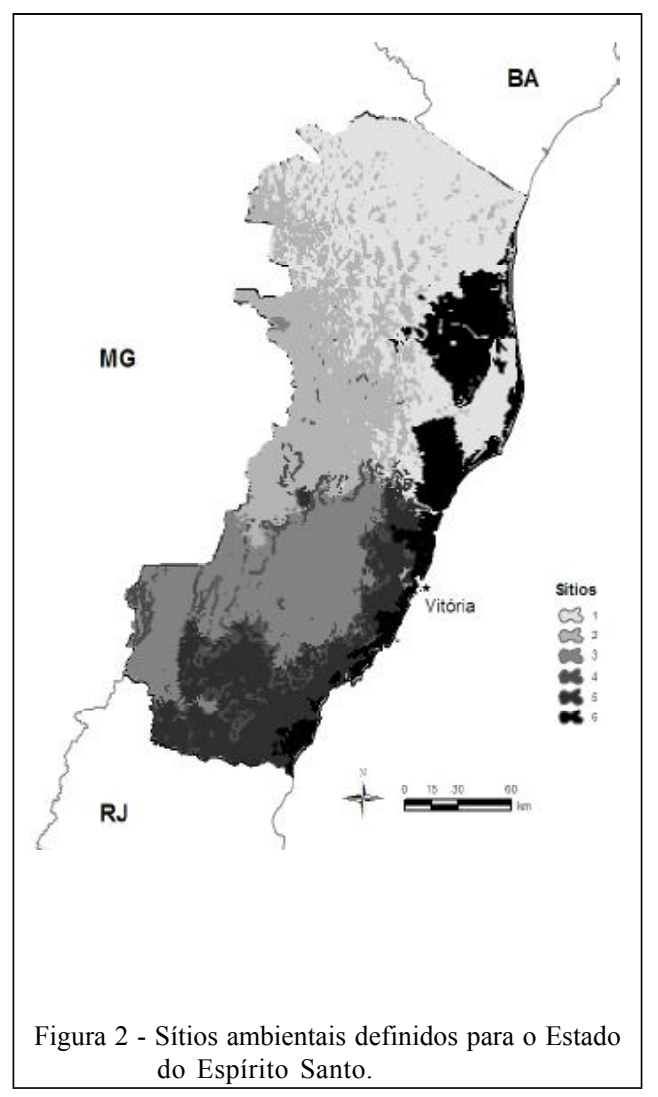

Ciência Rural, v.40, n.12, dez, 2010. 
Tabela 2 - Características físio-climaticas médias dos sítios ambientais delimitados.

\begin{tabular}{|c|c|c|c|c|c|c|}
\hline Variáveis & 1 & 2 & 3 & 4 & 5 & 6 \\
\hline Área $\left(\mathrm{km}^{2}\right)$ & 12880,36 & 10357,23 & 8241,909 & 2643,631 & 6404,652 & 5406,725 \\
\hline Altitude (m) & 169,5 & 370,48 & 883,7 & 678,09 & 246,28 & 60,93 \\
\hline Declividade (\%) & 1,16 & 4,5 & 4,27 & 16,41 & 4,16 & 0,48 \\
\hline Precipitação anual (mm) & 1147,67 & 1136,59 & 1379,47 & 1355,53 & 1355,28 & 1245,45 \\
\hline Excedente (mm) & 27,94 & 75,99 & 416 & 290,23 & 216,39 & 57,01 \\
\hline Deficiência (mm) & 285,47 & 246,87 & 39,4 & 77,53 & 81,89 & 198,61 \\
\hline Temperatura média anual $\left({ }^{\circ} \mathrm{C}\right)$ & 24,78 & 23,04 & 18,69 & 20,06 & 22,79 & 24,88 \\
\hline Amplitude térmica anual $\left({ }^{\circ} \mathrm{C}\right)$ & 20,28 & 20,12 & 19,77 & 20,02 & 20,2 & 20,33 \\
\hline ET potencial $(\mathrm{mm})$ & 1423,84 & 1312,64 & 1002,9 & 1144,08 & 1222,13 & 1398,6 \\
\hline ET real $(\mathrm{mm})$ & 1138,36 & 1065,76 & 963,5 & 1066,55 & 1140,23 & 1199,99 \\
\hline Fotoperíodo inverno (horas) & 10,87 & 10,84 & 10,76 & 10,76 & 10,74 & 10,81 \\
\hline Fotoperíodo verão (horas) & 13,12 & 13,15 & 13,23 & 13,23 & 13,25 & 13,17 \\
\hline DTA $(\mathrm{mm})$ & 0,19 & 0,21 & 0,21 & 0,19 & 0,20 & 0,11 \\
\hline
\end{tabular}

$\mathrm{Na}$ região litorânea centro-sul, foram encontrados os SAHs 5 e 6, com extensões próximas, ocupando 13,94 e 11,77\% da área do Estado, respectivamente. As diferenças topográficas foram influentes na diferenciação desses sítios. A DTA foi decisiva na delimitação do SAH 6, que apresentou o menor valor dessa variável. A presença de locais serranos no interior aumentou a altitude média local, levando a temperaturas médias ligeiramente menores no SAH 5. Relevante importância logística é verificada no SAH 6, em que boa parte do agronegócio capixaba e de outros estados é exportada pelos seus portos.

Diferentemente do que acontece nos trabalhos de zoneamento, onde a região de interesse se enquadra ou não às exigências climáticas de uma determinada cultura, neste trabalho, indiferente da cultura, foram definidas regiões com características edafoclimáticas semelhantes. A partir dessas áreas homogêneas, foram indicadas as possíveis culturas a serem implantadas, ou não, extraindo ao máximo o potencial de uso de cada uma dessas áreas.

Ao comparar os resultados encontrados em outros estudos realizados na mesma região, verificouse bom desempenho da metodologia utilizada nesta pesquisa. Em estudo de zoneamento para a espécie pinus (CASTRO et al., 2010), a região definida, segundo as exigências da cultura, foi praticamente a mesma que a da região homogênea 3 aqui delimitada. Portanto, a implantação do povoamento florestal ou de uma cultura agrícola pode ser direcionada a partir desses resultados.

\section{CONCLUSÕES}

Das variáveis climáticas utilizadas no estudo, as mais explicativas, após a rotação dos componentes, foram a amplitude térmica, a temperatura média e o ETR, explicando $85,3 \%$ da variância total acumulada.

As análises multivariada de agrupamento e a discriminante mostraram-se eficientes na delimitação e consistência dos sítios homogêneos, a partir dos parâmetros climáticos e topográficos, respectivamente.

Alguns sítios apresentaram similaridades climáticas, indicando que as variáveis topográficas utilizadas atuaram decisivamente na diferenciação deles.

\section{REFERÊNCIAS}

BERNARDO, S. et al. Manual de Irrigação. 7.ed. Viçosa: UFV, 2005. 611p.

CASTRO, F.S. et al. Zoneamento agroclimático para espécies do gênero Pinus no Estado do Espírito Santo. Floresta, v.40, n.1, p.235-250, 2010. Disponível em: <http://ojs.c3sl.ufpr.br/ ojs2/index.php/floresta/article/viewFile/17114/11274>. Acesso em: 03 jul. 2010.

DINPASHOH, Y. et al. Selection of variables for the purpose of regionalization of Iran's precipitation climate using multivariate methods. Journal of Hydrology, v.297, p.109123, 2004. . Disponível em: <http://www.sciencedirect.com/ science?_ob=ArticleURL\&_udi=B6V6C-4CHHRFM\&_user $=10 \& \_$coverDate $=09 \% 2 \mathrm{~F} 01 \% 2 \mathrm{~F} 2004 \& \_$rdoc $=1 \& \_\mathrm{fmt}$ 
$=$ high\&_orig $=$ search\&_sort $=\mathrm{d} \&$ docanchor $=\& \mathrm{view}=\mathrm{c} \&$ _search StrId $=1353100972 \&$ rerunOrigin $=$ google \&_acct $=\mathrm{C} 00005022$ $1 \&$ \&ersion $=1 \&$ urlVersion $=0 \&$ userid $=10 \& \mathrm{md} 5=0 \mathrm{e} 1 \mathrm{~b} 218 \mathrm{a} 05$ $7 \mathrm{~b} 8 \mathrm{c} 2 \mathrm{a} 3 \mathrm{c} 0 \mathrm{fac} 998 \mathrm{cc} 897>$. Acesso em: 15 ago. 2008. doi: 10.1016/j.jhydrol.2004.04.009.

GUILLEMETTE, N. et al. Feasibility study of a geostatistical modelling.of monthly maximum stream temperatures in a multivariate space. Journal of Hydrology, v.364, p.112, 2009. Disponível em: <http://www.sciencedirect.com/ science? ob=ArticleURL\& udi=B6V6C-4TPPF 83 $1 \&$ _user $=10 \&$ _coverDate $=01 \% 2 \mathrm{~F} 15 \% 2 \mathrm{~F} 2009 \&$ rdoc $=1 \&$ fm $\mathrm{t}=\mathrm{h}$ igh \& orig $=$ search\&_sort $=\mathrm{d} \&$ docanchor $=\& \mathrm{view}=\mathrm{c} \&$ _sea rchStrId $=1353110327 \&$ rerunOrigin $=$ google \&_acct $=\mathrm{C} 00005$ 0221 \&_version $=1 \&$ \&urlVersion $=0 \&$ \&userid $=10 \& \mathrm{md} 5=435290 \mathrm{f} 10 \mathrm{c}$ a798748426678a90c9afee. Acesso em: 15 ago. 2008. doi:10.1016/j.jhydrol.2008.10.002.

GRAEF, F. et al. Determining ecoregions for environmental and GMO monitoring networks. Environmental Monitoring and Assessment, v.108, p.189-203, 2005. Disponível em: <http:// www.springerlink.com/content/h40741877714q167/>. Acesso em: 12 jul. 2008. doi: 10.1007/s10661-005-3966-5.

HARTMANN, K.; WUNNEMANN, B. Hydrological hangesand Holocene climate variations in NW-China. Quaternary International, v.194, p.28-44, 2009. Disponível em: <http:/ / $\mathrm{w}$ w w. $\mathrm{s}$ c i e $\mathrm{n}$ c e d i r e c t . c o m/ science?_ob=ArticleURL\&_udi=B6VGS-4P8SJ9M$1 \&$ user $=10 \&$ _coverDate $=02 \% 2 \mathrm{~F} 01 \% 2 \mathrm{~F} 2009 \&$ rdoc $=1 \&$ f $\mathrm{mt}=$ high $\&$ orig $=$ search \&_sort $=\mathrm{d} \&$ _docanchor $=\& \mathrm{view}=\mathrm{c} \&$ _sear chStrId $=1353115704 \&$ rerunOrigin $=$ google\&_acct $=$ C000050221\&_ version $=1 \&$ \&urlVersion $=0 \& \_u s e r i d=10 \& \mathrm{md} \overline{5}=\mathrm{e} 293 \mathrm{~b} 3 \mathrm{aad} 9962 \mathrm{c} 4 \overline{\mathrm{d}}$ $1145 \mathrm{dd} 9373632 \mathrm{fe} 6>$. Acesso em: 16 ago. 2008. doi: doi:10.1016/j.quaint.2007.06.037.

IQBAL, M. Introduction to solar radiation. London:Academic Express, 1983. 212p.

INCAPER - Instituto Capixaba de Pesquisa, Assistência Técnica e Extensão Rural. Safra recorde. Disponível em: $<\mathrm{http}: / /$ www.incaper.es.gov.br/conteudo1.php>. Acesso em: 16 mar. 2008 .

LYRA, G.B. et al. Regiões homogêneas e funções de distribuição de probabilidade da precipitação pluvial no Estado de Táchira, Venezuela. Pesquisa Agropecuária Brasileira, v.41, p.205-215, 2006. Disponível em: <http:/ /www.scielo.br/scielo.php?script=sci_arttext\&pid=S0100204X2006000200004>. Acesso em: 15 ago. 2008. doi: 10.1590/S0100-204X2006000200004.

MACHADO, C.C. Colheita florestal. Viçosa: UFV, 2004. $468 \mathrm{p}$.

MORRIS, A.J. et al. Geospatial analysis of climatic and geomorphic interactions influencing stream discharge, Appalachian Mountains, USA. Environ Model Assess, v.14, p.73-84, 2009. Disponível em: <http://www.springerlink.com/ content/m841q2vgu25ujg9m/>. Acesso em: 10 jul. 2008. doi: 10.1007/s10666-008-9145-7.

ORTEGA, R.A.; SANTIBÁÑEZ, O.A. Determination of management zones in corn (Zea mays L.) based on soil fertility. Computers and Electronics in Agriculture, v.58, p.49-59, 2007. Disponível em: <http://portal.acm.org/ citation.cfm?id=1244808>. Acesso em: 18 ago. 2008 . doi:10.1016/j.compag.2006.12.011.

PEZZOPANE, J.E.M. et al. Espacialização da temperatura do ar no Espírito Santo. Revista Brasileira de Agrometeorologia, v.12, p.151-158, 2004. Disponível em: <http://www.scielo.br/ s cie 1 oOrg/php/reflinks.php? refpid = S 0100 $204 \times 200900040000100022 \&$ i d $=\mathrm{S} 0100$ 204X2009000400001\&lng=en>. Acesso em: 18 ago. 2008.

RAZIEI, T. at al. A precipitation-based regionalization forWestern Iran and regional drought variability. Hydrolgy and Earth System Sciences, v.12, p.1309-1321, 2008. Disponível em: <http://www.hydrol-earth-syst-sci.net/12/1309/ 2008/hess-12-1309-2008.html>. Acesso em: 16 ago. 2008. doi:10.5194/hess-12-1309-2008.

RHEE, J. et al. Delineation of climate regions using in-situ and remotely-sensed data for the Carolinas. Remote Sensing of Environment, v.112, p.3099-3111, 2008. Disponível em: $<\mathrm{http}$ :/ /www.sciencedirect.com/science?_ob=ArticleURL\&_udi=B6V6V$4 \mathrm{SBHF} 451$ \&_user $=10 \&$ coverDate $=06 \% 2 \mathrm{~F} 16 \% 2 \mathrm{~F} 2008 \&$ rdoc $=1 \&$ fmt $=$ high $\&$ orig $=$ search \&_sort $=\mathrm{d} \&$ docanchor $=\& \mathrm{vie} w=\mathrm{c} \&$ searchStrId $=1353125134 \&$ rerunOrigin $=$ google $\& \_$acct $=$C00005 $0221 \&$ version $=1 \&$ \&urlVersion $=0 \&$ userid $=10 \& \overline{m d} 5=692 \mathrm{~d} 004$ d9ebdeb069d3a02d04bdc0360>. Acesso em: 20 ago. 2008. doi:10.1016/j.rse.2008.03.001.

SEDIYAMA, G.C. et al. Modelo para estimativa das temperaturas normais mensais médias, máximas, mínimas e anual georreferenciados para o Estado do Espírito Santo. In: CONGRESSO BRASILEIRO ENGENHARIA AGRÍCOLA, 31., 2002, Salvador. Anais... Salvador: Sociedade Brasileira de Engenharia Agrícola, 2002. CD-ROM

THORNTHWAITE, C.W. An approach toward a rational classification of climate. Geographical Review, v.38, p.5594, 1948.

THORNTHWAITE, C.W., MATTER, J.R. The water balance. New Jersey: Publications in Climatology, 8, Centerton, 1955. 150p.

TIMILSENA, J.; PIECHOTA, T. Regionalization and reconstruction of snow water equivalent in the upper Colorado River basin. Journal of Hydrology, v.352, p.94106, 2008. Disponível em: <http://www.sciencedirect.com/ science? ob=ArticleURL\& udi=B6V6C-4RGM0V9$4 \&$ _user $=10 \&$ _coverDate $=04 \% 2 \mathrm{~F} 30 \% 2 \mathrm{~F} 2008 \&$ \& rdoc $=1 \&$ fmt $=$ high\&_orig $=$ search\&_sort $=$ d\&_docanchor $=\& v i e w=c$ 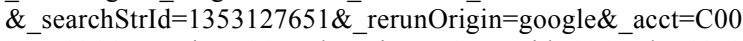 $0050221 \&$ version $=1 \&$ urlVersion $=0 \&$ userid $=10 \& \overline{m d} 5=22 \mathrm{e} 8$ $8 \mathrm{c} 688 \mathrm{ade} 9 \mathrm{c} 04 \mathrm{ce} 021 \mathrm{df} 71075 \mathrm{~cd} 4 \mathrm{~d}>$. Acesso em: 15 ago. 2008. doi:10.1016/j.jhydrol.2007.12.024. 